PROCEEDINGS OF THE

AMERICAN MATHEMATICAL SOCIETY

Volume 135, Number 6, June 2007, Pages 1875-1879

S 0002-9939(07)08674-1

Article electronically published on January 8, 2007

\title{
A NOTE ON A PROBLEM BY RICCERI ON THE AMBROSETTI-RABINOWITZ CONDITION
}

\author{
GIOVANNI ANELLO
}

(Communicated by David S. Tartakoff)

\begin{abstract}
In this note we give an answer to Ricceri's open problem involving the Ambrosetti-Rabinowitz superlinear condition.
\end{abstract}

\section{INTRODUCTION}

Throughout this note, $\Omega \subset \mathbb{R}^{N}$ is a nonempty open bounded set with sufficiently regular boundary $\partial \Omega$. Denote by $\mathcal{A}$ the set of all continuous functions $g: \mathbb{R} \rightarrow \mathbb{R}$ such that, if $N \neq 1$, there exists $q>0$ with $q<\frac{N+2}{N-2}$ if $N \geq 3$, satisfying

$$
\sup _{t \in \mathbb{R}} \frac{|g(t)|}{1+|t|^{q}}<+\infty \text {. }
$$

For each $c>2$ and $r \geq 0$ we put

$$
\mathcal{B}(r, c) \stackrel{\text { def }}{=}\left\{f \in \mathcal{A}: 0<c \int_{0}^{\xi} f(t) d t \leq \xi f(\xi) \text { for } \xi \in \mathbb{R} \backslash\{0\} \text { with }|\xi| \geq r\right\} .
$$

Note that what defines the set $\mathcal{B}(r, c)$ is the well-known Ambrosetti-Rabinowitz superlinear condition.

For every $f \in \mathcal{A}$ we put $\Phi_{f}(u)=\int_{\Omega}\left(\int_{0}^{u(x)} f(t) d t\right) d x$ for all $u \in W_{0}^{1,2}(\Omega)$. We equip $W_{0}^{1,2}(\Omega)$ with the usual norm $\|u\|=\left(\int_{\Omega}|\nabla u|^{2} d x\right)^{\frac{1}{2}}$. Let us consider the following subsets of $\mathcal{A}$ :

$$
\begin{aligned}
& \mathcal{C} \stackrel{\text { def }}{=}\left\{f \in \mathcal{A}: \inf _{\rho>0} \inf _{\|u\|<\rho} \frac{\sup _{\|v\|<\rho} \Phi_{f}(v)-\Phi_{f}(u)}{\rho^{2}-\|u\|^{2}}<\frac{1}{2}\right\}, \\
& \mathcal{D} \stackrel{\text { def }}{=}\left\{f \in \mathcal{A}: \lim _{t \rightarrow 0} \frac{f(t)}{t}=0\right\} .
\end{aligned}
$$

When $f \in \mathcal{D} \cap \mathcal{B}(r, c)$ for some $r \geq 0$ and $c>2$, the classical AmbrosettiRabinowitz result (see [1]) assures that the problem

$$
\begin{cases}-\Delta u=f(u) & \text { in } \Omega, \\ u=0 & \text { on } \partial \Omega\end{cases}
$$

Received by the editors December 5, 2005 and, in revised form, February 19, 2006. 2000 Mathematics Subject Classification. Primary 47J30.

(C)2007 American Mathematical Society Reverts to public domain 28 years from publication 1875 
has at least a non-zero weak solution in $W_{0}^{1,2}(\Omega)$. In recent years B. Ricceri has studied the above problem without assuming the condition $f \in \mathcal{D}$. In particular, he has established the following theorem.

Theorem A (Theorem 4 of [4]). Let $f \in \mathcal{B}(r, c)$ for some $r \geq 0$ and $c>2$. Then, for every $\rho>0$ and every

$$
\mu>\inf _{\|u\|<\rho} \frac{\sup _{\|v\|<\rho} \Phi_{f}(v)-\Phi_{f}(u)}{\rho^{2}-\|u\|^{2}}
$$

the problem

$$
\begin{cases}-\Delta u=\frac{1}{2 \mu} f(u) & \text { in } \Omega, \\ u=0 & \text { on } \partial \Omega\end{cases}
$$

admits at least two weak solutions in $W_{0}^{1,2}(\Omega)$, one of which has norm less than $\rho$.

A comparison between Theorem A and the Ambrosetti-Rabinowitz result leads to the following problem formulated by B. Ricceri in [3].

Problem. Does it hold the inclusion $\mathcal{B}(r, c) \subseteq \mathcal{C}$ for all $c>2$ and $r \geq 0$ ?

In this note we will prove that the inclusion in the above problem does not hold in general and that it holds only when $r=0$. Hence, in this latter case, we can remove the condition $f \in \mathcal{D}$ in the Ambrosetti-Rabinowitz result. Clearly the previous assertion makes sense only if we prove that there exists a function $f \in \mathcal{B}(0, c)$ which does not belong to $\mathcal{D}$. In our result we will prove the existence of such a function as well.

\section{The RESUlt}

The following theorem gives a complete answer to Ricceri's problem. We first point out that, by the proof, it will be clear that, if $N \geq 3$ and $c \geq \frac{2 N}{N-2}$, then $\mathcal{B}(r, c)=\emptyset$ for all $r \geq 0$.

Theorem 1. Let $c \in] 2, \frac{2 N}{N-2}[$ if $N \geq 3$ (resp. $c>2$ if $N=1,2$ ) and $r>0$. Then, one has:

i) $\mathcal{B}(r, c) \backslash \mathcal{C} \neq \emptyset$;

ii) $\mathcal{B}(0, c) \subseteq \mathcal{C}$;

iii) $\mathcal{B}(0, c) \backslash \mathcal{D} \neq \emptyset$.

Proof. We restrict ourselves to the case $N \geq 3$. The cases $N=1,2$ can be treated in the same manner with some slight modification. To verify i) fix $q \in] c-1, \frac{N+2}{N-2}[$. Define

$$
f(t)=a t+b|t|^{q-1} t,
$$

for all $t \in \mathbb{R}$, where $a$ is a positive real number satisfying

$$
\frac{1}{a}<\mu_{1}:=\sup _{u \in W_{0}^{1,2}(\Omega) \backslash\{0\}} \frac{\int_{\Omega}|u(x)|^{2} d x}{\|u\|^{2}}
$$

and

$$
b>a\left(\frac{c}{2}-1\right)\left(1-\frac{c}{q+1}\right)^{-1} r^{1-q} .
$$


From the definition of $f$, the inequalities in condition i) become

$$
0<\frac{c a}{2} \xi^{2}+b \frac{c}{q+1}|\xi|^{q+1} \leq a \xi^{2}+b|\xi|^{q+1}
$$

which are satisfied if $|\xi| \geq r$, taking the choice of $b$ into account. Consequently, we have $f \in \mathcal{B}(r, c)$. Let us to show that $f \notin \mathcal{C}$. In view of Theorem 2.1 of [2], it suffices to check that the functional

$$
\Psi(u)=\frac{1}{2}\|u\|^{2}-\Phi_{f}(u),
$$

$u \in W_{0}^{1,2}(\Omega)$, has no local minima. In fact, if $v \in W_{0}^{1,2}(\Omega)$ was a local minimum for $\Psi$, then, taking into account that $\Psi$ is of class $C^{2}$ (this latter fact comes out from the condition $q>1$ ), we should have

$$
\Psi^{\prime \prime}(v)(u, u) \geq 0
$$

for all $u \in W_{0}^{1,2}(\Omega)$. Hence

$$
\int_{\Omega}|\nabla u(x)|^{2} d x-a \int_{\Omega}|u(x)|^{2} d x-q \int_{\Omega}|v(x)|^{q-1}|u(x)|^{2} d x \geq 0
$$

for all $u \in W_{0}^{1,2}(\Omega)$. In particular, choosing $u=v_{1}$, where $v_{1}$ is an eigenvector related to the first eigenvalue of the laplacian $\lambda_{1}=\frac{1}{\mu_{1}}$, we should have, being $a \mu_{1}>1$,

$$
\begin{aligned}
0= & \int_{\Omega}\left|\nabla v_{1}(x)\right|^{2} d x-\frac{a}{a \mu_{1}} \int_{\Omega}\left|v_{1}(x)\right|^{2} d x \\
& >\int_{\Omega}\left|\nabla v_{1}(x)\right|^{2} d x-a \int_{\Omega}\left|v_{1}(x)\right|^{2} d x-q \int_{\Omega}|v(x)|^{q-1}\left|v_{1}(x)\right|^{2} d x \geq 0
\end{aligned}
$$

which is absurd.

To prove ii) let $f \in \mathcal{B}(0, c)$. Then, there exists $q \in] 1, \frac{N+2}{N-2}[$ such that

$$
M:=\sup _{t \in \mathbb{R}} \frac{|f(t)|}{1+|t|^{q}}<+\infty .
$$

Hence,

$$
0<\int_{0}^{\xi} f(t) d t \leq M\left(|\xi|+\frac{1}{q+1}|\xi|^{q+1}\right)
$$

for all $\xi \in \mathbb{R}$. At this point, consider the real function

$$
g(\xi)=\frac{\int_{0}^{\xi} f(t) d t}{|\xi|^{c}}
$$

defined for all $\xi \in \mathbb{R} \backslash\{0\}$. One has $g \in C^{1}(\mathbb{R} \backslash\{0\})$ and

$$
g^{\prime}(\xi)=\frac{|\xi|^{c} f(\xi)-c|\xi|^{c-2} \xi \int_{0}^{\xi} f(t) d t}{|\xi|^{2 c}}=\frac{\xi^{2} f(\xi)-c \xi \int_{0}^{\xi} f(t) d t}{|\xi|^{c+2}}
$$

for all $\xi \in \mathbb{R} \backslash\{0\}$. So, from the fact that $f \in \mathcal{B}(0, c)$, we easily deduce that $g$ is positive, decreasing in $\mathbb{R}_{-}$and increasing in $\mathbb{R}_{+}$. Then, for a suitable positive number $L$, we have

$$
\int_{0}^{\xi} f(t) d t \leq L|\xi|^{c}
$$


for all $\xi \in \mathbb{R}$ with $|\xi| \leq 1$. Now, from (1) we can find a positive constant $M_{1}$ such that

$$
\int_{0}^{\xi} f(t) d t \leq M_{1}|\xi|^{q+1}
$$

for all $\xi \in \mathbb{R} \backslash[-1,1]$. Hence, putting together (2) and (3), we have

$$
\int_{0}^{\xi} f(t) d t \leq L|\xi|^{c}+M_{1}|\xi|^{q+1}
$$

for all $\xi \in \mathbb{R}$. At this point, from (4), for suitable positive constants $C_{1}, C_{2}$ we have

$$
\begin{aligned}
& \frac{\sup _{\|u\| \leq \rho} \Phi_{f}(u)}{\rho^{2}} \leq \frac{\sup _{\|u\| \leq \rho} \int_{\Omega}\left(L|u(x)|^{c}+M_{1}|u(x)|^{q+1}\right) d x}{\rho^{2}} \\
& \leq \frac{\sup _{\|u\| \leq \rho} C_{1}\|u\|^{c}+C_{2}\|u\|^{q+1}}{\rho^{2}}=C_{1} \rho^{c-2}+C_{2} \rho^{q-1} \rightarrow 0
\end{aligned}
$$

as $\rho \rightarrow 0^{+}$. This implies $f \in \mathcal{C}$.

We finally prove iii). Let $\left.c_{1} \in\right] c, \frac{2 N}{N-2}\left[\right.$ and $p=\log _{2}\left(\frac{c_{1}}{c_{1}-c}\right)$. Put

$$
f(t)=|t|^{c_{1}-2} t+g(t) t
$$

for all $t \in \mathbb{R}$, where

$$
g(t)= \begin{cases}\frac{\left(t-2^{-n}\right)\left(2^{-n}+2^{-\left(n c_{1}+p\right)}-t\right)}{2^{-2\left(n c_{1}+p+1\right)}} & \text { if } n \in \mathbb{N}, t \in\left[2^{-n}, 2^{-n}+2^{-\left(n c_{1}+p\right)}\right] \\ 0 & \text { otherwise }\end{cases}
$$

By an easy calculation we see that

$$
\lim _{n \rightarrow+\infty} \frac{f\left(2^{-n}+2^{-\left(n c_{1}+p+1\right)}\right)}{2^{-n}+2^{-\left(n c_{1}+p+1\right)}}=1
$$

then $f \notin \mathcal{D}$. On the other hand, one has $f \in \mathcal{A}$. Now, let $\xi \neq 0$. It is easily seen that

$$
\int_{0}^{\xi} f(t) d t>0
$$

Moreover, if $\xi<0$, then we have

$$
\xi f(\xi)-c \int_{0}^{\xi} f(t) d t=\left(1-\frac{c}{c_{1}}\right)|\xi|^{c_{1}}>0 .
$$


While, if $\xi>0$, denoting by $n$ the small positive integer such that $2^{-n}<\xi$, we have

$$
\begin{aligned}
\xi f(\xi) & -c \int_{0}^{\xi} f(t) d t \\
& \geq \frac{c_{1}-c}{c_{1}}|\xi|^{c_{1}}-c \sum_{k=n}^{\infty} \int_{2^{-k}}^{2^{-\left(k c_{1}+p\right)}} t \frac{\left(t-2^{-k}\right)\left(2^{k}+2^{-\left(k c_{1}+p\right)}-t\right)}{2^{-2\left(k c_{1}+p+1\right)}} \\
& \geq \frac{c_{1}-c}{c_{1}}|\xi|^{c_{1}}-\frac{c}{6} \sum_{k=n}^{\infty} 2^{-\left(k c_{1}+p\right)}\left(2^{-(k-1)}+2^{-\left(k c_{1}+p\right)}\right) \\
& \geq \frac{c_{1}-c}{c_{1}} 2^{-n c_{1}}-2^{-p} \frac{c}{3} \sum_{k=n}^{\infty} 2^{k c_{1}}=2^{-n c_{1}}\left(\frac{c_{1}-c}{c_{1}}-2^{-p} \frac{c}{3\left(1-2^{\left.-c_{1}\right)}\right.}\right) \\
& \geq 2^{-n c_{1}}\left(\frac{c_{1}-c}{c_{1}}-2^{-p}\right)=0 .
\end{aligned}
$$

Consequently, $f \in \mathcal{B}(0, c)$. The proof is now complete.

Remark. From the above proof it is easy to check that, in point of fact, the following inclusion holds:

$$
\left\{f \in \mathcal{A}: 0<c \int_{0}^{\xi} f(t) d t \leq \xi f(\xi) \text { for } \xi \in \mathbb{R} \backslash\{0\} \text { with }|\xi| \leq r\right\} \subseteq \mathcal{C}
$$

for all $r>0$ and $c>2$.

\section{REFERENCES}

[1] A. Ambrosetti, P. Rabinowitz, Dual variational methods in critical point theory and applications, J. Funct. Anal. 14 (1973), 349-381. MR0370183 (51:6412)

[2] B. Ricceri, A general variational principle and some of its applications, J. Comput. Appl. Math. 113 (2000), 401-410. MR1735837 (2001h:47114)

[3] B. Ricceri, Some research perspectives in nonlinear functional analysis, Seminar on fixed point theory, Cluj-Napoca 3 (2002), 99-110. MR1929750 (2003g:47107)

[4] B. Ricceri, On a classical existence theorem for nonlinear elliptic equations, in "Esperimental, constructive and nonlinar analysis", M. Théra ed., 275-278, CMS Conf. Proc. 27, Canad. Math. Soc. (2000). MR1777629 (2001h:35068)

Department of Mathematics, University of Messina, 98166 S. Agata, Messina, Italy

E-mail address: anello@dipmat.unime.it 